\title{
MODELING AND CONTROL OF A DIESEL HCCI ENGINE
}

\author{
J. Chauvin* A. Albrecht* G. Corde* N. Petit ${ }^{* *}$ \\ * Institut Français du Pétrole, 1 et 4 Avenue de Bois \\ Préau, 92852 Rueil Malmaison, France \\ jonathan. chauvin@ifp.fr \\ ** Centre Automatique et Systèmes, École des Mines de \\ Paris, \\ 60, bd St Michel, 75272 Paris, France
}

\begin{abstract}
This article focuses on the control of a Diesel engine airpath. We propose a detailed description of the airpath of a Diesel HCCI engine supported by experimental results. Moreover, we propose a simple, yet innovative, motion planning control strategy. At the light of this study, we can finally conclude, with supportive results, that motion planning is indeed an appropriate solution for controlling the airpath dynamics.
\end{abstract}

Keywords: Diesel, airpath control, modeling, non minimum phase

\section{INTRODUCTION}

Performance and environmental requirements of Diesel engines have steadily increased over the last thirty years, which in turn has required an increase in the sophistication of employed control strategies. Advances in model based control over this period have been one of the keys in meeting the demands placed on modern combustion technologies. Lately, the Highly Premixed Combustion mode (HPC) - including Homogeneous Charge Compression Ignition (HCCI) - has become of major interest. It requires the use of high Exhaust Gas Recirculation (EGR) rates. The key idea is that the recirculated burned gas lower the in-cylinder temperature and dilute the air charge which reduces the emissions of nitrogen oxides. Simultaneous ignition in the whole combustion chamber is performed and controlled. Significant reduction in pollutants emission was proven in practice through numerous experiments (see (Kahrstedt et al., 2003; Amnéus et al., 1998) for example). In that combustion mode, the core variable is the Burned Gas Rate (BGR) in the intake manifold (see Figure 1). BGR offsets may cause misfires and malicious noises. In the HCCI combustion mode it is very high ( $40 \%$ or more). Accurate control of BGR can be achieved by controlling the whole airpath system: intake and exhaust manifolds, EGR loop and fresh air loop. This is the subject of the paper.

As studied in (Kolmanovsky et al., 1997; Kao and Moskwa, 1995b), the airpath system of a turbocharged Diesel engine features coupled dynamics. The EGR acts as a discharge valve for the turbocharger. Most studies consider the following control setup: both intake pressure and intake air flow are closely controlled using EGR valve and Variable Geometry Turbocharger (VGT) using Gain schedulling PI controllers as in (Stefanopoulou et al., 2000; van Nieuwstadt et al., 2000), using constructive Lyapunov control as in (Janković and Kolmanovsky, 2000) or LPV formulation as in (Jung and Glover, 2005). Controlling both intake and exhaust pressure has been exposed in (Ammann et al., 2003). All these studies prove the relevance of a multivariable control. 
Following a preliminary approach we presented in (Chauvin et al., 2006), we use a motion planning strategy by expliciting a feed-forward term.

The contribution of the paper is as follows. We propose a detailed description of the airpath of a Diesel HCCI engine supported by experimental results. Moreover, we propose a simple, yet innovative, motion planning control strategy. At the light of this study, we can finally conclude, with supportive results, that motion planning is indeed an appropriate solution for controlling the airpath dynamics.

The paper is organized as follows. In Section 2, we detail the control problem. In Section 3, we detail the influence of the airpath actuators on the combustion process. Section 4 proposes a simplified model for control purpose. We explicit the control strategy and experimental results on a 4 cylinder HCCI engine in Section 6. Conclusions and future directions are given in Section 7 .

\section{CONTROL PROBLEM}

Our approach to combustion control is to manage the air and burned gas masses in the cylinder. In other words, we focus on the airpath system. Figure 1 shows the flow sheet of the airpath. Flows of fresh air and the Exhaust Gas Recirculation (EGR) mix into the intake manifold and are aspirated into the cylinders.

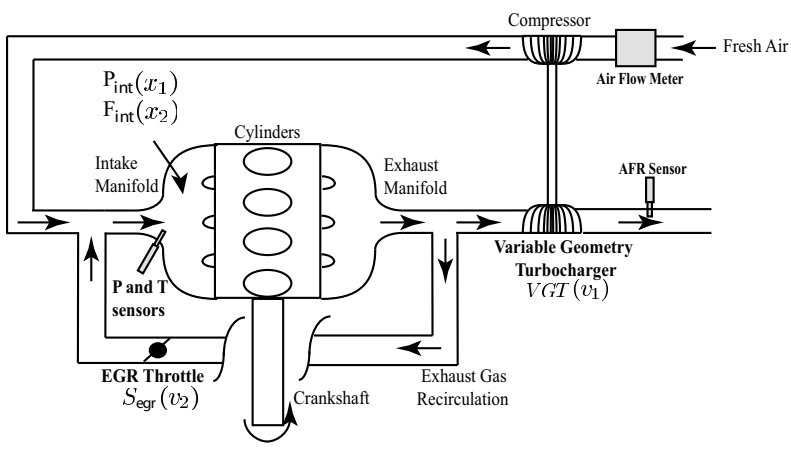

Figure 1. Air path problem. The sensors used are colored in grey.

Engine control performance requirements usually include consumption, pollutants, and noise reduction. Those requirements are often incompatible. Therefore, a tradeoff is needed. This impacts the reference setpoints of air and burned gas masses in the cylinder $\left(M_{a i r, c y l}\right.$ and $\left.M_{b g, c y l}\right)$. In practical applications, those variables can not be measured. Yet, equivalent variables can be considered. Controlling those two masses is equivalent to controlling the intake pressure $P_{\text {int }}$ (being an image of $\left.M_{a i r, c y l}+M_{b g, c y l}\right)$ and the burned gas rate $F_{\text {int }}$ (representing $\frac{M_{b g, c y l}}{M_{a i r, c y l}+M_{b g, c y l}}$ ). Setpoints are often chosen to maximize EGR in order to lower the $\mathrm{NO}_{x}$ emissions at low load. At high load, the negative impact of EGR onto combustion stability and efficiency imposes the use of low EGR. Typically, the setpoint at $1500 \mathrm{rpm}$ and high load is $\left(P_{\text {int }, s p}=2 \mathrm{bar}, F_{\text {int }, s p}=.05\right)$ using low EGR, while at $1500 \mathrm{rpm}$ and low load setpoints under consideration are close to $\left(P_{\text {int }, s p}=\right.$ 1.013bar, $\left.F_{\text {int }, s p}=.45\right)$ using high EGR.

In this context, the control problem we need to address is a large transient problem for a two outputs, two inputs system. The control inputs are the VGT actuator position $S_{v g t}\left(v_{1}\right)$ (ranging from 0 to 1 ) and the EGR valve normalized effective area $S_{\text {egr }}\left(v_{2}\right)$ (ranging from 0 to 1 ). Both are bounded. Other external inputs include the fueling rate and the engine speed $N_{e}$. The underlying dynamics is also of dimension 2 . The states are the outputs: $P_{\text {int }}$ and $F_{\text {int }}$.

\section{INFLUENCE ON THE COMBUSTION PROCESS}

The air feedback loop purpose is to control the gas mixture in the cylinder, i.e. the air and burned gas masses in this case. Unfortunately, these variables can not be measured directly on the engine. Yet, relied variables can be considered as the fresh air mass flow $D_{\text {air }}$, the intake pressure $P_{\text {int }}$, the intake temperature $T_{i n t}$ and the burned gas rate in the intake manifold $F_{\text {int }}$. In a classical Diesel HCCI engine architecture, two actuators are available to control the air path : the EGR valve located on the EGR loop and the Variable Geometry Turbine (VGT) distributor. Since the air and EGR paths are strongly coupled, it is not possible to deal with the two actuators independently with two separated control loops :

- To vary the fresh air mass flow rate, the corresponding energy required by the turbine from the exhaust gas depends on the available mass flow, which is directly linked with the EGR flow.

- To vary the EGR flow, the corresponding pressure deviation required between the exhaust and the intake manifolds is directly linked to the turbocharger operating conditions (supercharging pressure and exhaust counter-pressure).

The next subsections describe with more details the actuator influence on the air path and the intake condition impact on the combustion process.

\subsection{Actuator influence on the air path}

Influence of the EGR valve actuator Basically, the EGR valve regulates the mass flow rate of gas 
coming from the exhaust manifold to the intake manifold. At a fixed EGR valve position, the EGR flow is established according to the pressure deviation between intake and exhaust manifolds. Opening the EGR valve increases the EGR flow but decreases the available energy for the turbine and may decrease both the intake pressure $P_{\text {int }}$ and the fresh air mass flow $D_{\text {air }}$. The influence of the EGR valve position on the air path at fixed operating conditions is represented in Figure 2. A summary of the impact of the EGR valve on the gas aspirated in the cylinder is reported in Table 1.
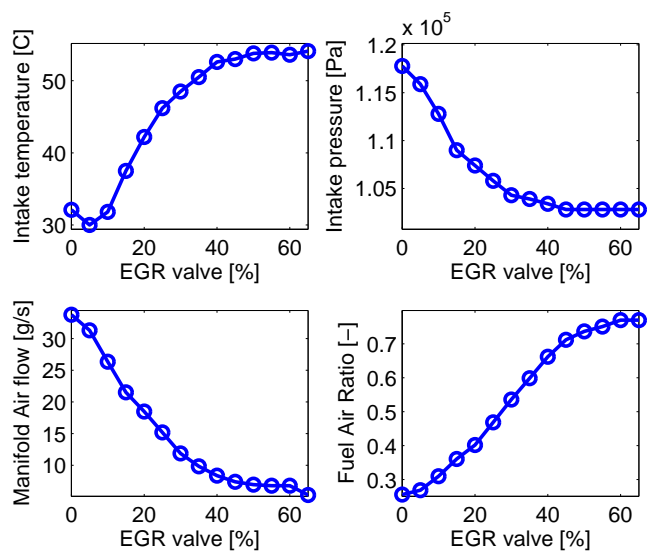

Figure 2. Experimental variation of the EGR valve at fixed operating conditions (4 bar IMEP injection strategy, $N_{e}=1500 \mathrm{rpm}, \mathrm{VGT}=$ $20 \%$ ). Effect on the air path.

\begin{tabular}{|l||c|c|c|}
\hline Variable & $M_{a s p, \text { air }}$ & $M_{a s p, b g}$ & $M_{a s p}$ \\
\hline EGR valve $\uparrow$ & $\downarrow$ & $\uparrow$ & $\downarrow$ \\
\hline VGT $\uparrow$, low EGR & $\downarrow$ & $\uparrow$ & $\downarrow$ \\
\hline VGT $\uparrow$, high EGR & $\uparrow$ & $\downarrow$ & $*$ \\
\hline
\end{tabular}

Table 1. Summary of the impact of the actuators at fixed operating conditions. $\uparrow$ increase, $\downarrow$ decrease, $*$ minor effect.

Influence of the turbocharger actuator Basically, the VGT regulates the supercharging pressure which is directly related to the fresh air mass flow $D_{\text {air }}$ and intake pressure $P_{\text {int }}$. Under fixed operating conditions, closing the VGT increases the part of the energy available at the turbine housing intake that will go through the turbine wheel and, so, increase the turbocharger speed and then the compression ratio of the compressor. Therefore, it causes the increase of the intake manifold pressure. At the same time, closing the VGT increases the exhaust counter-pressure due to the turbine and so, the exhaust manifold pressure. Both intake and exhaust manifold pressures are then modified with the same trend because of the VGT modification. This potential modification of the pressure deviation between intake and exhaust manifolds may impact the EGR flow but with a variable trend. Because of this interaction with the EGR loop, the effect of the
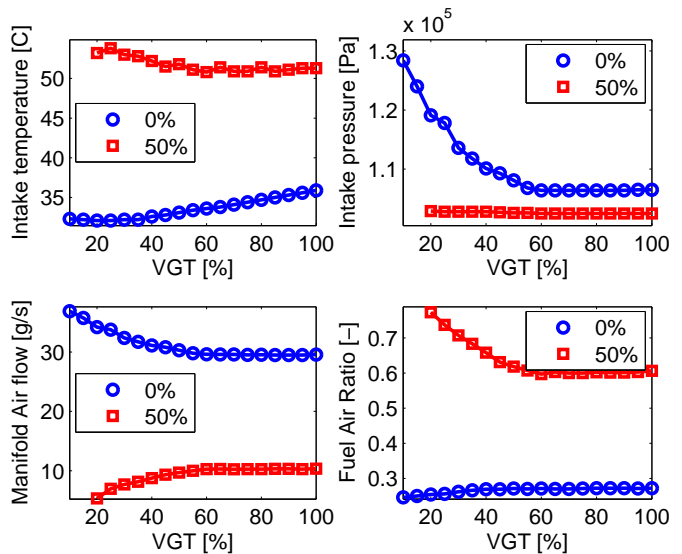

Figure 3. Experimental variation of the VGT actuator at fixed operating conditions (4 bar IMEP injection strategy, $N_{e}=1500 \mathrm{rpm}$, EGR valve $=0 \%$ and $50 \%$ ). Effect on the air path.

VGT on the steady state values of $D_{\text {air }}$ is not always monotonic. For the HCCI Diesel engine, this is especially difficulty to manage when the EGR valve is largely open. In this case, closing the VGT to increase the fresh air mass flow may lead to decrease $D_{\text {air }}$. The influence of the VGT for two EGR valve positions at fixed operating conditions is presented in Figure 3. A summary of the impact of the VGT on the gas aspirated in the cylinder is reported in Table 1.

\subsection{Intake condition impact on the combustion process}

In the previous subsection, we described the impact of the actuators on the air path variables (intake pressure, intake temperature, ...). Now, we comment the impact on the combustion through the pressure curve. For that, we look at several combustion parameter:

- $P_{c y l, m a x}$ : the maximum cylinder pressure during the combustion.

- $C A_{50}$ : crank Angle when $50 \%$ of the fuel has been burnt.

- $\tau_{a i}$ : auto-ignition delay.

- Noise: noise produced during the combustion (correlated to the maximum of pressure gradient).

- IMEP: torque produced by the combustion.

According to the BGR level, to introduce burned gases in the combustion chamber modifies the combustion process in several ways :

- It plays the role of an inert gas that increases the cylinder gas mixture thermal inertia : this is the main effect at low BGR.

- It plays the role of a third body that enhances the apparition of specific chemical reactions 
(catalytic effect) and slows down the reaction rates (diluting effect) : this is the main effect at high BGR.

The BGR has therefore a great impact on the combustion process. In an HCCI Diesel engine, it participates to reduce the temperature peak, to increase the global auto-ignition delay of the main combustion, to have the cold flame phenomenon, and finally to reduce the $\mathrm{NO}_{x}$ production. Note that it is the total burned gas fraction in the cylinder that acts on the combustion. These burned gas are comprised of both residual gas from the previous cycle and exhaust gas cooled by the EGR cooler and recycled to the intake. Figure 4 presents the cylinder pressures obtained from an EGR valve variation at the engine testbed. A great impact can be noticed on the combustion process and on the engine response in terms of $\mathrm{NO}_{x}$ emission and noise production as presented in the Figure 5. Unfortunately, this figure also shows that the BGR is not the only intake conditions that has varied during these tests and the intake pressure and temperature have also been modified. In fact, it is very difficult to obtain a single intake condition variation from experimentation. That is one of the reasons why the engine simulation support is very useful for control development (Albrecht et al., 2006b). In this case, a phenomenological combustion chamber model has been used to simulate the combustion process. After being validated on a wide range of operating conditions, the model has been used to perform single intake condition variations. More details about the phenomenological modelling approaches used in this study and the $\mathrm{HCCI} /$ conventional combustion model validation are presented in (Albrecht et al., 2006a).

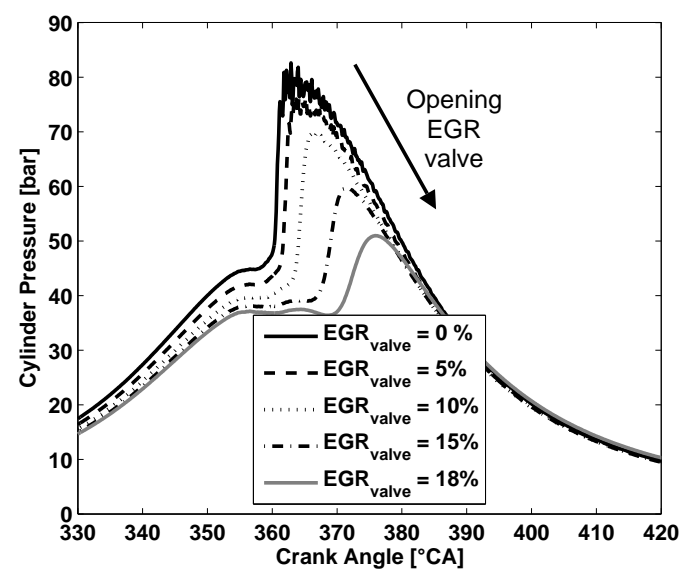

Figure 4. Experimental variation of the EGR valve at fixed operating conditions (5 bar IMEP injection strategy, $N_{e}=1500 \mathrm{rpm}, \mathrm{VGT}=$ $33 \%)$. Effect on the cylinder pressure.

Based on this combustion model, single intake condition variation simulations have been per-
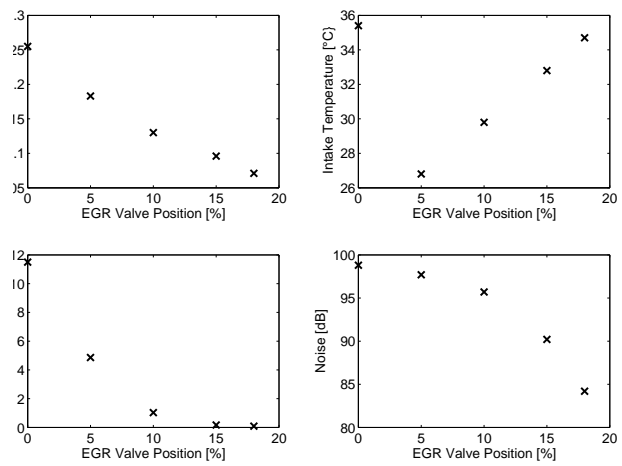

Figure 5. Experimental variation of the EGR valve at fixed operating conditions (5 bar IMEP injection strategy, $N_{e}=1500 \mathrm{rpm}$, VGT $=$ $33 \%)$. Effect on the intake pressure, intake temperature, $\mathrm{NO}_{x}$ and noise versus BGR.

formed for BGR, pressure intake and temperature intake around the reference operating set point of 5 bar IMEP and $1500 \mathrm{rpm}$ engine speed.Increasing the BGR leads to increase the main combustion start angle by modifying the auto-ignition delay and enhancing the cold flame phenomenon. Thanks to simulation support, this BGR effect can be observed more accurately on the burned mass fraction $(\mathrm{BMF})$ results which allows in particular to identify the cold flame phenomenon (linear part of the BMF curve). Moreover, the increase of the mixture thermal inertia coupled with the delayed combustion start after the top dead center angle decreases the maximum cylinder pressure. In Figure 6, it can be observed that increasing the intake pressure leads to a higher pressure at the top dead center angle and then speeds up the ignition occurrence and increases the maximum cylinder pressure. The intake temperature variation has a lower effect on the cylinder pressure, influencing lightly the auto-ignition delay. This variable is not taken into account in this paper but can be introduced later since it can be useful in the context of the HCCI combustion high sensitivity. A summary of the impact of the air path variables is reported in Table 2.

\begin{tabular}{|l||c|c|c|c|c|}
\hline Variable & $P_{c y l, \max }$ & $C A_{50}$ & $\tau_{A I}$ & Noise & IMEP \\
\hline$F_{\text {int }} \uparrow$ & $\downarrow$ & $\uparrow$ & $\uparrow$ & $\downarrow$ & $\downarrow$ \\
\hline$P_{\text {int }} \uparrow$ & $\downarrow$ & $\downarrow$ & $\downarrow$ & $\uparrow$ & $\downarrow$ \\
\hline$T_{\text {int }} \uparrow$ & $*$ & $\downarrow$ & $\downarrow$ & $\uparrow$ & $*$ \\
\hline
\end{tabular}

Table 2. Summary of the impact of the air path elements. $\uparrow$ increase, $\downarrow$ decrease, * minor effect.

\section{INTAKE MANIFOLD MODELLING}

Flows of fresh air (measured by the Manifold Air Flow) and from the EGR come into the 


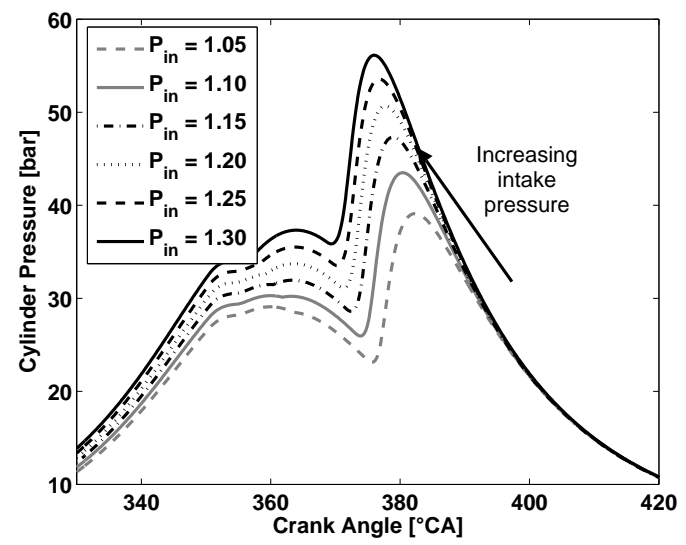

Figure 6. Numerical variation of the intake pressure at fixed in-cylinder conditions ( 5 bar IMEP injection strategy, $\mathrm{Ne}=1500 \mathrm{rpm}$ reference set point). Effect on the cylinder pressure.

intake manifold to be aspirated into the cylinders. In numerous references found in the literature (e.g. (Tsai and Goyal, 1986; Kao and Moskwa, $1995 a)$ ), mean value engine modelling approaches are considered. These use temporal and spatial averages of relevant temperatures, pressures and mass flow rates, and lead to a seven state reference model. The states are the intake and exhaust manifold pressure, temperature, and composition, and the turbocharger speed. Further, we propose a reduction down to 2 states. Motivations are given in the following subsection.

\subsection{Modelling assumptions}

First of all, as it is standard in modelling the intake manifolds in spark-ignited engines (e.g. (Hendricks et al., 1996)), we neglect the temperature fluctuations. Out of the seven usual state variables, two are eliminated. The already discussed common model reduction (see (van Nieuwstadt et al., 1998; Stefanopoulou et al., 2000), (Jung and Glover, 2005) and (Janković and Kolmanovsky, 2000)) consists of a three dimensional reference control model using the intake pressure, the exhaust pressure and the turbocharger speed as states. Composition dynamics are not taken into account because the corresponding two states (intake and exhaust composition) are difficult to measure and are only weakly observable from the remaining three states. In our case, we use the Air/Fuel Ratio sensor located downstream the turbine. It is an image of the composition in the exhaust manifold. This major difference with usually considered setups suggests us to substitute the exhaust pressure dynamics with the intake composition dynamics. Finally, we neglect the turbocharger dynamics. The reason for this simplification is that the turbocharger speed dynamics is very slow compared to the pressure and composition dynamics. Finally, the turbocharger speed is modelled as a static function of the intake pressure $P_{\text {int }}$ and the intake air flow $D_{\text {air }}$. These hypothesis yield a reduction down to a two dimensional state.

\subsection{State space model}

Two balance equations provide the model we build our work on.

Total mass balance in the intake manifold Ideal gas law in the intake manifold leads to

$$
P_{\text {int }} V_{\text {int }}=M_{\text {int }} R T_{\text {int }}
$$

Assuming that variations of temperature are small, mass balance writes

$$
\dot{P}_{i n t}=\frac{R T_{i n t}}{V_{i n t}}\left(D_{a i r}+D_{e g r}-D_{a s p}\right)
$$

Classically (see (Heywood, 1988) for example), we assume that the aspirated flow can be computed as $D_{a s p}=\eta_{v o l}\left(P_{i n t}, N_{e}\right) \frac{P_{i n t}}{R T_{i n t}} V_{c y l} \frac{N_{e}}{120}$ where $V_{c y l}$ is the cylinder volume. $\eta_{v o l}$ is the volumetric efficiency which is experimentally derived and, eventually, defined though a look-up table $\eta_{\text {vol }}\left(P_{\text {int }}, N_{e}\right)$.

Composition balance in the intake manifold The burned gas ratio $F_{\text {int }}$ is the fraction of burned gas in the intake manifold. It writes

$$
F_{i n t} \triangleq 1-\frac{M_{i n t, a i r}}{M_{i n t}}
$$

The composition of the EGR $\left(F_{\text {egr }}\right)$ is the composition in the exhaust manifold $\left(F_{\text {exh }}\right)$ delayed by the transport through the EGR pipe. We consider that this delay is negligible, i.e. $F_{\text {egr }}=F_{\text {exh }}$. Mixing dynamics is modelled as

$$
\dot{F}_{i n t}=\frac{R T_{i n t}}{P_{i n t} V_{i n t}}\left(D_{e g r}\left(F_{e x h}-F_{i n t}\right)-D_{a i r} F_{i n t}\right)
$$

Gathering (1) and (2) leads to the following reference system

$$
\left\{\begin{array}{l}
\dot{x}_{1}=\alpha_{i n t}\left(u_{1}+u_{2}-\eta_{v o l}\left(x_{1}, N_{e}\right) \beta_{i n t} x_{1}\right) \\
\dot{x}_{2}=\frac{\alpha_{i n t}}{x_{1}}\left(F_{\text {exh }} u_{2}-\left(u_{1}+u_{2}\right) x_{2}\right)
\end{array}\right.
$$

\section{INTAKE DYNAMICS ISSUES}

As described in (Kolmanovsky et al., 1997; Kao and Moskwa, 1995b), the coupled air path dynamics features nonminimum phase dynamics on some operating points. 


\subsection{Step of VGT position}

When applying a closing step in the VGT control signal, the exhaust manifold pressure rises. This results in a higher mass flow through the EGR and a higher intake manifold pressure. Closing the VGT also results in lower mass flows through both the turbine and the compressor. But, as a result of the increasing pressure in the exhaust manifold, these mass flows eventually increase and, after a while, reach a state where the mass flows are higher than before the step in the VGT position. This is a typical behavior for a nonminimum phase behavior. It results of zeros in the right half plane in the pole-zero map of the system.

\subsection{Step in EGR position}

When the EGR valve opens, first the flow through the EGR valve increases thereby increasing the intake manifold pressure. However, since a relatively smaller portion of the exhaust gas is supplied to the turbocharger, and since the exhaust manifold is emptied at a higher rate when the EGR valve opens, eventually the intake manifold pressure decreases. The latter effect is, however, delayed because of the turbocharger dynamics.

\subsection{Non minimum phase behavior}

Around a steady state point $(\bar{x}, \bar{u})$, the linearization of (3) leads to $\dot{\delta x}=A \delta x+B \delta u$, where $\delta x \triangleq$ $x-\bar{x}, \delta u \triangleq u-\bar{u}, A \triangleq-\alpha_{\text {int }}\left[\begin{array}{cc}\beta_{\text {int }} & 0 \\ 0 & \frac{\bar{u}_{1}+\bar{u}_{2}}{\bar{x}_{1}^{2}}\end{array}\right]$ and $B \triangleq \alpha_{i n t}\left[\begin{array}{cc}1 & 1 \\ -\frac{\bar{x}_{2}}{\bar{x}_{1}} & \frac{F_{e x h}-\bar{x}_{2}}{\bar{x}_{1}}\end{array}\right]$. If we apply a step of input $\delta u$, we have

$\dot{\delta x}(0)=\alpha_{\text {int }}\left[\begin{array}{c}\delta u_{1}(0)+\delta u_{2}(0) \\ \frac{F_{e x h} \delta u_{2}(0)-\bar{x}_{2}\left(\delta u_{1}(0)+\delta u_{2}(0)\right)}{\bar{x}_{1}}\end{array}\right]$

Then, asking more EGR and more pressure will lead to a high increase of $\delta u_{1}+\delta u_{2}$ leading to an inverse response of the BGR.

\section{EXPERIMENTAL RESULTS}

\subsection{Motion planning control strategy}

Yet, using (appropriately valued) step inputs does not provide satisfactory transients. It has been impossible for us to get such a control strategy
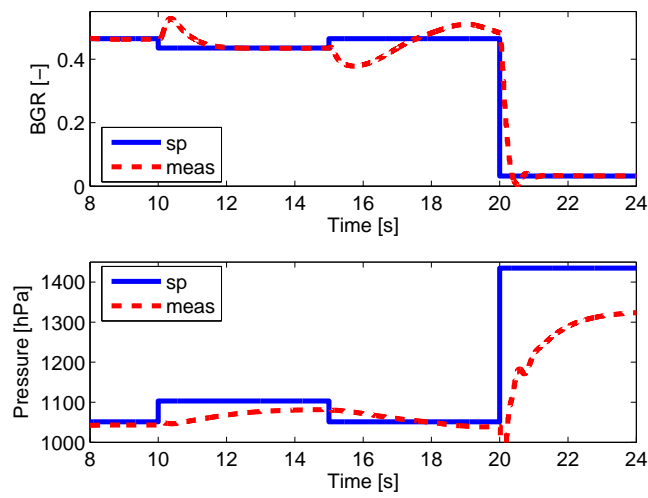

Figure 7. Tentative open loop transients with step inputs.

to work efficiently on our test bench. Numerical simulations through the AMESIM environment have provided us with some insights. In Figure 7, it appears that without model-based feedforward action, one is not able to take into account the apparent non-minimum phase effect of dynamics (3) (as seen in (Kolmanovsky et al., 1997) for example). There are too many oscillations and too large errors. These can not be damped before a new transient is to be achieved.

On the other hand, it also appears that interaction between air and EGR loops combined with the nonlinear nature of the system between highly varying setpoints makes the system difficult to handle using classical control design methods. We propose a motion planning control strategy which rely on the computation of transient trajectories for the airpath dynamics (3). This strategy is detailed in (Chauvin, 2006). It comprises 4 sub procedures: setpoint computations through static maps, trajectory generation, model inversion, and saturation of open-loop control values. We explicit the main part, the model inversion, in the next subsection.

\subsection{Model inversion}

System (3) is fully actuated and invertible. Thus, an analytic expression of the input can be derived from the state variables and their first derivatives histories. In fact,

$$
\left\{\begin{array}{c}
u_{1}+u_{2}=\eta_{v o l}\left(x_{1}, N_{e}\right) \beta_{i n t} x_{1}+\frac{1}{\alpha_{i n t}} \dot{x}_{1} \\
-x_{2} u_{1}+\left(F_{\text {exh }}-x_{2}\right) u_{2}=\frac{1}{\alpha_{\text {int }}} \dot{x}_{2} x_{1}
\end{array}\right.
$$

This rewrites

$$
\left\{\begin{array}{l}
u_{1}=f_{1}(x, \dot{x}) \\
u_{2}=f_{2}(x, \dot{x})
\end{array}\right.
$$

with 


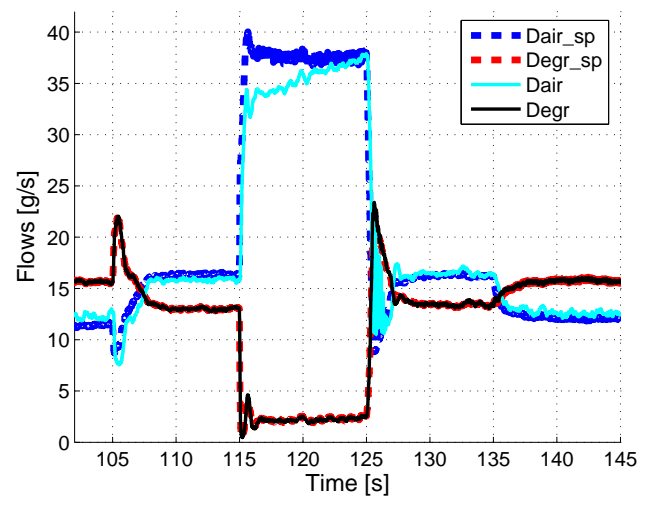

Figure 8. Experimental results: IMEP transient from 2 to 5 bar, to 9 bar, to 5 bar, and then to 4 bar at $1500 \mathrm{rpm}$. Flow histories. Dashed : set point $\left(u^{\mathrm{sp}}\right)$, solid: closed-loop trajectory.

$$
\left\{\begin{aligned}
f_{1}(x, \dot{x})= & \frac{1}{F_{\text {exh }}}\left(\frac{F_{\text {exh }}-x_{2}}{\alpha_{\text {int }}} \dot{x}_{1}-\frac{1}{\alpha_{\text {int }}} \dot{x}_{2} x_{1}\right. \\
& \left.+\left(F_{\text {exh }}-x_{2}\right) \eta_{\mathrm{vol}}\left(x_{1}, N_{e}\right) \beta_{\text {int }} x_{1}\right) \\
f_{2}(x, \dot{x})= & \frac{1}{F_{\text {exh }}}\left(\frac{1}{\alpha_{\text {int }}} x_{2} \dot{x}_{1}+\eta_{\mathrm{vol}}\left(x_{1}, N_{e}\right) \beta_{\text {int }} x_{2} x_{1}\right. \\
& \left.+\frac{1}{\alpha_{\text {int }}} \dot{x}_{2} x_{1}\right)
\end{aligned}\right.
$$

In these last expressions, $F_{\text {exh }}, \alpha_{\text {int }}, N_{e}$, and $\beta_{\text {int }}$ are all given by sensors measurements. The unique open-loop control law $\left(u_{1}^{\mathrm{mp}}, u_{2}^{\mathrm{mp}}\right)$ corresponding to any desired $\left(x_{1}^{\mathrm{mp}}, x_{2}^{\mathrm{mp}}\right)$ trajectory is

$$
\left\{\begin{array}{l}
u_{1}^{\mathrm{mp}}=f_{1}\left(x_{1}^{\mathrm{mp}}, \dot{x}_{1}^{\mathrm{mp}}, x_{2}^{\mathrm{mp}}, \dot{x}_{2}^{\mathrm{mp}}\right) \\
u_{2}^{\mathrm{mp}}=f_{2}\left(x_{1}^{\mathrm{mp}}, \dot{x}_{1}^{\mathrm{mp}}, x_{2}^{\mathrm{mp}}, \dot{x}_{2}^{\mathrm{mp}}\right)
\end{array}\right.
$$

\subsection{Experimental results}

Figures 8 and 9 report experimental closed-loop results. The scenario is a varying torque demand at constant engine speed (1500 rpm) in both HCCI combustion mode and conventional combustion mode. We now detail these experiments.

From $t=102 s$ to $t=112 s$

here, we have an IMEP transient at $1500 \mathrm{rpm}$ in HCCI combustion mode. The IMEP of the system starts at 2 bar and eventually reaches 5 bar. This transient aims higher intake pressure and BGR setpoints. Starting and ending operating points are both in HCCI combustion mode. Let us focus on Figure 8. By contrast with all decentralized controllers, we notice on that our controller takes into account the well known non minimum phase behavior of the system reported in (Kolmanovsky et al., 1997). More precisely, one can check that the main contribution to this is due to the openloop controller (the closed loop control histories being very close to it). When the EGR valve

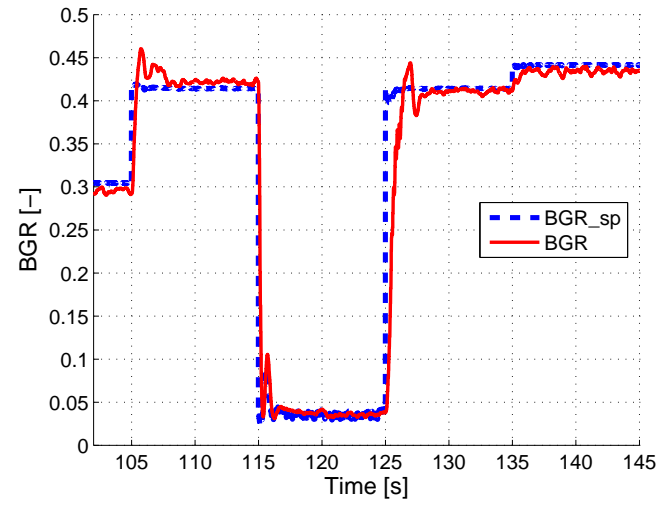

Figure 9. Experimental results: IMEP transient from 2 to 5 bar, to 9 bar, to 5 bar, and then to 4 bar at $1500 \mathrm{rpm}$. BGR histories. Dashed : set point, solid: closed-loop trajectory.

opens, the flow increases leading to a pressure rise in the intake manifold. Meanwhile, the exhaust pipe acts as a discharge for the VGT. Its opening lowers the EGR supplied to the turbocharger yielding a significant drop of the exhaust manifold flow. The turbocharger slows down which eventually causes the decrease of the intake manifold pressure. This phenomenon is delayed and slowed down by the turbocharger inertia. Simple ramps and/or steps will fail to let the system reach the desired setpoint. With the proposed control strategy, the model takes into account this complex behavior. The motion planning efficiently drives the system to its setpoint.

From $t=112 \mathrm{~s}$ to $t=122 \mathrm{~s}$

here, we have a tip-in (high increase of torque demand) at $1500 \mathrm{rpm}$. Implicitly, it is desired to steer the system from a low load point with high EGR to a high load point with much less BGR. The proposed open loop control strategy successively closes the EGR valve and then closes the VGT with an overshoot. One can notice the resulting decrease in EGR flow and simultaneous increase of the fresh air flow. As expected from a motion planning control strategy, this does provide a soft landing for the state variables $x_{1}$ and $x_{2}$ onto their set points. During the transient, the open loop control laws are indeed saturated. This results in the temporary mismatch between the airflow and its set point. This effect is particularly noticeable on this very large pressure transient that we choose for sake of illustration.

Again, transients are smooth and present only small oscillations. It is instructive to note that, in this exact same setup, we failed to get a decentralized controller preventing both stall and noises. The main reason for this seems the undesired overshoot of the BGR. 


\section{CONCLUSION}

The presented work demonstrates the relevance of motion planning in the control of the -coupledairpath dynamics of turbocharged Diesel engines using Exhaust Gas Recirculation. For the HCCI combustion mode, very large rates of burned gas need to be considered and we have proven on a realistic test-bench cases that the proposed approach can handle such situations. Despite strong coupling, the airpath dynamics has nice properties that make it easy to steer through our control strategy. Its triangular form yields exponential convergence over a wide range of setpoints. It can also be shown, through a simple analysis, to satisfy operational constraints, provided transient are chosen sufficiently smooth.

The next step will be to adapt the strategy to other engine configurations, i.e. two turbochargers and/or two EGR pipes with various thermal conditions.

Acknowledgments The authors would like to thank Yann Creff for his support and Dominique Soleri for providing the experimental facilities.

\section{REFERENCES}

Albrecht, A., J. Chauvin, F.-A. Lafossas, S. Potteau and G. Corde (2006a). Development of highly premixed combustion Diesel engine model : from simulation to control design. In: Proc. of the SAE Conference. number 200601-1072.

Albrecht, A., P. Moulin, O. Grondin and G. Corde (2006b). Control design for hcci combustion engine based on virtual engine simulator: from control development to real-time calibration. In: Proc. of THIESEL Conference.

Ammann, M., N. Fekete, L. Guzzella and A. Glattfelder (2003). Model-based control of the VGT and EGR in a turbocharged commonrail Diesel engine: theory and passenger car implementation. In: Proc. of the SAE Conference. number 2003-01-0357.

Amnéus, P., D. Nilsson, F. Mauss, M. Christensen and B. Johansson (1998). Homogeneous Charge Compression Ignition engine: experiments and detailed kinetic calculations. In: $4^{\text {th }}$ International Symposium on Diagnostic and Modeling in Internal Combustion Engines.

Chauvin, J. (2006). Estimation and Control of a Diesel HCCI engine. Estimation for time periodic systems. PhD thesis. École des Mines de Paris.

Chauvin, J., G. Corde, N. Petit and P. Rouchon (2006). Experimental motion planning in air- path control for HCCI engine. In: Proc. of the American Control Conference.

Hendricks, E., A. Chevalier, M. Jensen, S. Sorenson, D. Trumpy and J. Asik (1996). Modelling of the intake manifold filling dynamics. In: Proc. of the SAE Conference.

Heywood, J. (1988). Internal Combustion Engine Fundamentals. McGraw-Hill, Inc.

Janković, M and I. Kolmanovsky (2000). Constructive Lyapounov control design for turbocharged Diesel engines. Proc. of the IEEE Control Systems Technology 8, 288-299.

Jung, M. and K Glover (2005). Comparison of uncertainty parameterisations for H-infinity robust control of turbocharged Diesel engines. Proc. of Control Engineering Practice 13, 1525.

Kahrstedt, J., K. Behnk, A. Sommer and T. Wormbs (2003). Combustion processes to meet future emission standards. In: Motortechnische Zeitschrift. pp. 1417-1423.

Kao, M. and J. Moskwa (1995a). Turbocharged Diesel engine modeling for nonlinear engine control and estimation. Proc. of the ASME Journal of Dynamic Systems, Measurements and Control.

Kao, M. and J. Moskwa (1995b). Turbocharged Diesel engine modelling for nonlinear engine control and estimation. Proc. of the ASME Journal of Dynamic Systems, Measurements and Control.

Kolmanovsky, I., A. Stefanopoulou, P. Moraal and M. van Nieuwstadt (1997). Issues in modelling and control of intake flow in variable geometry turbocharged engines. In: Proc of the $18^{\text {th }}$ IFIP Conference on System Modelling and Optimization.

Stefanopoulou, A., I. Kolmanovsky and J. Freudenberg (2000). Control of variable geometry turbocharged Diesel engines for reduced emissions. Proc. of the IEEE Control Systems Technology 8, 733-745.

Tsai, S.-C. and M. Goyal (1986). Dynamic turbocharged Diesel engine model for control analysis and design. In: Proc. of the SAE Conference.

van Nieuwstadt, M., I. Kolmanovsky, P. Moraal, A. Stefanopoulou and M. Janković (2000). Experimental comparison of EGR-VGT control schemes for a high speed Diesel engine. Proc. of the IEEE Control Systems Magazine 20, 63-79.

van Nieuwstadt, M., P. Moraal, I. Kolmanovsky, A. Stefanopoulou, P. Wood and M. Criddle (1998). Decentralized and multivariable designs for EGR-VGT control of Diesel engine. In: Proc. of the IFAC Symp. Advances in Automotive Control. 